\title{
DIWATA-2 TARGETING ASSESSMENT AND ATTITUDE ERROR DETERMINATION USING A QUATERNION-BASED TRANSFORMATION SYSTEM
}

\author{
K. Monay ${ }^{1 *}$, F.R. Olivar ${ }^{1}$, B.J. Magallon ${ }^{1}$, M.E.A. Tupas ${ }^{1}$ \\ ${ }^{1}$ GRASPED, STAMINA4Space Program, University of the Philippines Diliman, Quezon City, Philippines \\ (krmonay, fmolivar) @ stamina4space.upd.edu.ph, bpmagallon@gmail.com, matupas@up.edu.ph
}

\section{Commission IV}

KEY WORDS: targeting assessment; quaternions; transformation system; attitude; vector rotation

\begin{abstract}
:
Target pointing assessment of a space-borne satellite is vital to its operations especially on microsatellites that have limited camera field of view and attitude control components like in the case of Diwata-2. In this study, two scientific payloads of the satellite were used: the Enhanced Resolution Camera (ERC) with a field of view (FoV) of $89.8 \times 67.5 \mathrm{~km}$ and a resolution of 54.6m; and the High Precision Telescope (HPT) with a FoV of $3.1 \times 2.3 \mathrm{~km}$ and a resolution of $4.7 \mathrm{~m}$. Errors in pointing especially on a payload with a small field of view like the HPT could mean the satellite missing its target. The target pointing of Diwata-2 is assessed by firstly, computing the differences in the coordinates of the planned target, the center of the actual image taken by the satellite and the projected target from the satellite's attitude logs. As such, a quaternion-based transformation system is created to simulate the satellite's local vertical local horizontal system from a given Earth-centered inertial system. Secondly, the differences were then tabulated, and its averages were computed to derived pointing corrections. Applying the algorithm to the satellite's images shows that there is an average error in pitch and roll of $0.590^{\circ}$ and $0.004^{\circ}, 6.436^{\circ}$ and $6.503^{\circ},-5.8465^{\circ}$ and $-6.499^{\circ}$ between the set target to the actual image acquired, between the actual image and from attitude logs and between the set target and from the attitude logs, respectively.
\end{abstract}

\section{INTRODUCTION}

The PHL-Microsat Program launched its second microsatellite, Diwata-2, last October 29, 2018 at an altitude of $600 \mathrm{~km}$. The microsatellite aims to monitor vegetation, assess damages and provide a way for communication during and after disasters. The Diwata-2 is equipped with four payloads similar to the preceding satellite, Diwata-1: the Space-borne Multispectral Imager with Liquid Crystal Tunable Filter (SMI-LCTF) with a ground sampling distance (GSD) of $126.9 \mathrm{~m}$ and a field of view (FoV) of 83.7 x $62.7 \mathrm{~km}$; the High Precision Telescope (HPT) with a GSD of $4.7 \mathrm{~m}$ and a FoV of 3.1 x $2.3 \mathrm{~km}$; the Wide Field Camera (WFC); and the Middle Field Camera (MFC), which is an engineering payload with a GSD of $287.2 \mathrm{~m}$ and a FoV of 489.3 $\mathrm{x} 141.9 \mathrm{~km}$. Two additional payloads were installed to help fulfill its objectives and to provide better service to the users. To improve the spatial resolution of the SMI-LCTF payload, the microsatellite is equipped with the Enhanced Resolution Camera (ERC), a panchromatic camera with a GSD of $54.6 \mathrm{~m}$ and a FoV of 89.8 x $67.5 \mathrm{~km}$. To provide communication during disasters, an amateur radio unit is also installed with two modes: FM voice repeater mode and APRS digital voice repeater mode.

Since the microsatellites are mainly used for remote sensing purposes, the data it provides must be handled and calibrated well. Thus, there is a need for the satellite to capture its target. Currently, there are three general pointing modes for the Diwata2: the nadir, off-nadir, and target mode. Diwata- 2 enters its nadir mode when it takes images directly below the satellite as it moves in the orbit. Off-nadir pointing happens when the satellite tilts and captures images at an angle from the nadir as the satellite moves. Lastly, target pointing happens when the satellite fixed its target in a set point location in the ground as it moves along the orbit in order to take an image with the target in it. Magallon, et al (2018) defines the pointing modes for Diwata-1, which is the same for Diwata-2.
Currently, Diwata-2 records its roll, pitch and yaw values that would help determine the image centers of the observation. Magallon, et al (2018) explains the Attitude Determination and Control System (ACDS) of the Diwata-1; and it applies as well to the Diwata-2. The ACDS determines and controls the satellite's attitude and pointing mode. A GPS module determines the satellite's position and velocity vectors. Attitude is determined through Coarse and Fine attitude determination. The former uses the relative illumination of the sun to the satellite (Sun Aspect Sensor) and the magnetic field intensity around the satellite (Geomagnetic Aspect Sensor) to determine the satellite's attitude. The latter uses a photo of the stars in its field of view and a star map as reference for the captured photo (Magallon, et al, 2018). These attitude values are then projected into the Earth thus determining the image centers.

After determining the satellite's attitude, its error's effect on the pointing of the satellite should be known. Pitch errors generally introduce errors along the trajectory. Roll errors, on the other hand, introduce errors perpendicular to the trajectory. Yaw errors introduce the image orientation error. The latter introduces few to small changes with respect to the center of the image recorded by the satellite. Thus, this study was conducted to determine the current attitude errors, specifically pitch and roll, of the sensors of Diwata-2 in order to execute its necessary calibration to obtain better data quality to achieve its purpose. Since the microsatellites are not subject to maintenance, knowing the attitude errors from the target to the actual image is necessary so future targets are adjusted depending on the acquired errors.

Magallon, et al (2018) determined the attitude errors of the Diwata-1 images. These errors were attributed to the sun azimuth, sun elevation, and the Earth's magnetic field due to their effects on the Sun and Geomagnetic Aspect Sensors. The average pointing error of the Diwata-1 is 20 kilometers. For the purposes of this paper, the error along the trajectory will be defined as timing error, as this theoretically can be fixed by 
applying corrections to the timing of acquisition. The error along the line perpendicular to the trajectory will be defined as the pointing error.

This study uses three coordinate systems to determine the pitch and roll angles of the satellite to a point on the Earth. The EarthCentered Inertial (ECI) system considers the Earth's center of mass and its primary vectors fixed for the celestial sphere (Rummel, Peters, 2001). This system is primarily used to determine the position of satellites with respect to the celestial sphere. As such, Diwata-2's position and velocity vectors use the ECI system. The paper also uses the Earth-Centered, Earth-Fixed (ECEF) system which considers the Earth's center of mass but with its primary vectors fixed with the Earth (Rummel, Peters, 2001). This system differs from the ECI system due to the latter considering the motion of the celestial sphere relative to Earth. Thus, the rotation and position of the planet on a given date and time is considered for the position of an object in an ECI system. $\mathrm{ECEF}$, on the other hand, do not consider the motion of the Earth and its positions are independent of the time and date. As such, geodetic coordinates of points such as targets and image centers are to be converted from the ECEF system to ECI. Lastly, the Local Vertical, Local Horizontal (LVLH) system is the satellite's local coordinate system with one primary vector parallel to the satellite's orbit path and another to its nadir. This system is helpful in determining the satellite's pitch and roll values for the study.

Conversion to different coordinate system require rotations of vectors. The most common parameters of representing vector rotation are the direction cosine matrix, Euler angles and quaternions.

Direction cosine matrix (DCM) describes a rotation through direction cosine values from its initial coordinate system to its target coordinate system. These direction cosine values are derived from the cosine of the angles between the vector and the three coordinate axes. Euler angles describes a 3D rotation through a sequence of $2 \mathrm{D}$ rotations from about each of the three coordinate axes. (Aimati, 2015)

Quaternions describe a rotation by a rotational angle from a rotational axis which is not necessarily around and about the three coordinate axes like the Euler angles, it rotates a vector around an inertial coordinate system. (Yang, 2011; Perumal, 2011). A quaternion is composed of four components, 1 real component and 3 imaginary components. It can be expressed as:

$$
Q=q+q_{0}
$$

where $\mathrm{Q}=$ quaternion

$\mathrm{Q}=$ real component/scalar component

$\mathrm{q}_{\mathrm{o}}=$ imaginary component

The imaginary component $\left(\mathrm{q}_{\mathrm{o}}\right)$ can be further expressed through 3 imaginary axes $(\mathrm{i}, \mathrm{j}, \mathrm{k})$.

$$
q_{0}=q_{1} \hat{\imath}+q_{2} \hat{\jmath}+q_{3} \hat{k}
$$

where $\mathrm{q}_{1}, \mathrm{q}_{2}, \mathrm{q}_{3}$ are scalar values.

Rotation described through quaternions is expressed in a single equation:

where

$$
T=Q \times t \times Q^{*}
$$

$\mathrm{T}=$ rotated vector

$$
\mathrm{Q}=\text { quaternion }
$$

$$
\begin{aligned}
& \mathrm{t}=\text { vector in the inertial coordinate system } \\
& \mathrm{Q}^{*}=\text { conjugate quaternion }
\end{aligned}
$$

The conjugate quaternion is defined as:

$$
Q^{*}=q-q_{0}
$$

where $\quad q=$ real component/scalar component

$\mathrm{q}_{\mathrm{o}}=$ imaginary component

Quaternion based methods has been studied for its application in rotation (Perumal, 2011) and for its application in spacecraft attitude determination and control (Yang, 2011). Several advantages of using quaternions compared to other types of conversion were also stated in these studies. Quaternions are more compact and executes less computation compared to DCM. (Perumal, 2011) Through quaternion, controllers can globally stabilize nonlinear spacecraft system while in using Euler Angles may not stabilize such spacecrafts because it uses a linear model (Yang, 2011).

With Diwata-1 nearing the end of its lifespan, it is imperative to assess the targeting errors of the Diwata-2 to give more images capturing the intended targets. Thus, the main objective of the study is to create a working transformation system to determine attitude values from the satellite's position and velocity vectors, the coordinates of the targets, and the date and time of observation, and the calibration values for target setting.

\section{METHODOLODY AND RESULTS}

\subsection{Methodology}

Several missions were conducted to assess the current attitude of Diwata-2 images. Images were captured in different places that were in the path of the microsatellite with different pointing methods; nadir, off-nadir, and target pointing. These images were then used to determine the pointing and timing errors by determining the distance between the image center, attitude log, and the target location. The timing error is the distance between the image center and the target along the path of the microsatellite. The pointing error is the distance between the image center and the target along the line perpendicular to the path of the microsatellite.

To determine the attitude errors, it is necessary to convert the different coordinate systems to the satellite's LVLH system. The satellite's position and velocity vectors come from its installed GPS module, and is in the ECI coordinate system, with its reference vectors be $(1,0,0),(0,1,0)$ and $(0,0,1)$. These vectors were then used to create the satellite's LVLH coordinate system by making the position vector the Z-axis. Cross-multiplying the $\mathrm{Z}$-axis to the satellite's velocity vector creates a vector (X-axis, or the cross-track axis) perpendicular to the velocity vector. Finally, multiplying the $\mathrm{Z}$-axis and the $\mathrm{X}$-axis gives the $\mathrm{Y}$-axis or the along-track axis of the satellite.

The images used were data products of Diwata-2's Enhanced Resolution Cameras (ERC) and the red sensor of its HPT (HPT$\mathrm{R})$. The image centers were converted from latitude and longitude values (ECEF system) into the ECI system, using its capture date.

In this study, three targets are to be determined. The programmed target refers to the target coordinates uploaded to the satellite. The projected target refers to the target coordinates projected by 
the satellite's pitch, roll and yaw values at the time of image capture. Lastly, the actual target is the center of the image captured by the satellite. The programmed target is important as this is the location the user wants to capture. As such, the errors in targeting will be referred to the programmed target. Defining the errors in the projected target is also helpful as this determines the status and capability of the satellite to record accurate values of its attitude.

The differences in coordinates between the targets (both actual, projected and programmed) and the satellite position vectors were then transformed into the satellite's LVLH system by applying a quaternion. Since the reference axes of the ECI and the LVLH systems are already defined, it is necessary to determine the quaternion converting the ECI coordinate system to LVLH. Besl, McKay (1992) defines a method of extracting transformation parameters between two sets of points by minimizing a mean square objective function during registration. These transformation parameters contain an axis to where the points are to be rotated and the angle of rotation. These parameters can be expressed as a quaternion. After extraction, the vectors from the satellite position to the targets are then converted by applying equation 3 .

Finally, given the difference in position in the new coordinate system, the pitch and roll values were determined by taking the angle forwards and backwards, or sideward, respectively, from the satellite position to the target.

To check the values of the code, the position and path of the Diwata-2 is determined through the Satellite Tool Kit (STK) software and the measurements of the timing and pointing errors are compared to the projection of the attitude errors by multiplying their tangents to the altitude of the Diwata-2.

\subsection{Results and Discussion}

Tables 1 and 2 list the pitch and roll values (in decimal degrees) for the targets and the image centers while tables 3 and 4 shows the pitch and roll error values (in decimal degrees) for the ERC and HPT-R, respectively. For tables 3 and 4, the first column shows the area captured and the pointing system used. The second column shows the error between the programmed target and the actual target. The third column shows the error between the projected target and the actual image center. The last column shows the difference between the programmed target and the target projected by the recorded attitude values of the satellite. Positive pitch values denote forward tilt while positive roll values denote a counterclockwise tilt. For missions with targeting mode, the pitch and roll values are the average of the entire mission.

\begin{tabular}{|c|c|c|c|}
\hline Mission & $\begin{array}{c}\text { Programmed } \\
\text { Target }\left(^{\circ}\right)\end{array}$ & $\begin{array}{c}\text { Projected } \\
\text { Target }\left(^{\circ}\right)\end{array}$ & $\begin{array}{c}\text { Image } \\
\text { Center }\left(^{\circ}\right)\end{array}$ \\
\hline $\begin{array}{c}\text { Tripoli, } \\
\text { Lebanon } \\
\text { (Nadir) }\end{array}$ & 6.4567 & -0.1776 & 8.0858 \\
& 0.5111 & -0.1503 & 1.2810 \\
\hline $\begin{array}{c}\text { Dubai, UAE } \\
\text { (Nadir) }\end{array}$ & 5.5129 & -0.0025 & 7.0549 \\
\hline $\begin{array}{c}\text { Muscat, } \\
\text { Oman } \\
\text { (Off-nadir) }\end{array}$ & 3.4457 & -0.1507 & 0.8714 \\
\hline
\end{tabular}

\begin{tabular}{|c|c|c|c|}
\hline $\begin{array}{c}\text { Manila, } \\
\text { Philippines } \\
\text { (Target) }\end{array}$ & 25.2317 & 25.7990 & 27.4897 \\
\hline Damascus, & 13.4081 & 13.4666 & 14.5864 \\
Syria \\
(Target)
\end{tabular}

Table 1. Pitch/Roll Values for ERC

\begin{tabular}{|c|c|c|c|}
\hline Mission & $\begin{array}{l}\text { Programmed } \\
\text { Target }\left(^{\circ}\right)\end{array}$ & $\begin{array}{l}\text { Projected } \\
\text { Target }\left({ }^{\circ}\right)\end{array}$ & $\begin{array}{c}\text { Image } \\
\text { Center }\left(^{\circ}\right)\end{array}$ \\
\hline $\begin{array}{l}\text { Tripoli, } \\
\text { Lebanon } \\
\text { (Nadir) }\end{array}$ & $\begin{array}{l}6.4567 \\
0.5111\end{array}$ & $\begin{array}{l}-0.1776 \\
-0.1503\end{array}$ & $\begin{array}{l}8.0630 \\
1.2206\end{array}$ \\
\hline $\begin{array}{l}\text { Dubai, UAE } \\
\text { (Nadir) }\end{array}$ & $\begin{array}{l}5.5129 \\
0.2457\end{array}$ & $\begin{array}{l}-0.0025 \\
-0.1507\end{array}$ & $\begin{array}{l}7.0324 \\
0.8179\end{array}$ \\
\hline $\begin{array}{l}\text { Muscat, } \\
\text { Oman } \\
\text { (Off-nadir) }\end{array}$ & $\begin{array}{l}4.4742 \\
3.4061\end{array}$ & $\begin{array}{l}-2.0884 \\
0.4753\end{array}$ & $\begin{array}{l}4.1567 \\
3.1493\end{array}$ \\
\hline $\begin{array}{c}\text { Damascus, } \\
\text { Syria } \\
\text { (Target) }\end{array}$ & $\begin{array}{l}3.2783 \\
0.0018\end{array}$ & $\begin{array}{l}-4.3894 \\
-25.2250\end{array}$ & $\begin{array}{c}1.9311- \\
1.7493\end{array}$ \\
\hline
\end{tabular}

Table 2. Pitch/Roll Values for HPT-R

\begin{tabular}{|c|c|c|c|}
\hline Mission & $\begin{array}{c}\text { Pitch/Roll } \\
\text { Error } \\
\text { (Act - Prog) }\end{array}$ & $\begin{array}{c}\text { Pitch/Roll } \\
\text { Error } \\
\text { (Act - Proj) }\end{array}$ & $\begin{array}{c}\text { Pitch/Roll } \\
\text { Error } \\
\text { (Proj - Prog) }\end{array}$ \\
\hline $\begin{array}{c}\text { Tripoli, } \\
\text { Lebanon }\end{array}$ & $\begin{array}{c}1.6291 \\
0.7698\end{array}$ & $\begin{array}{l}8.2635 \\
1.4312\end{array}$ & $\begin{array}{l}-6.6343 \\
-0.6614\end{array}$ \\
\hline Dubai, UAE & 1.5420 & 7.0573 & -5.5154 \\
& 0.6257 & 1.0220 & -0.3963 \\
\hline Muscat, & -0.3596 & 6.2030 & -6.5626 \\
Oman & -0.3928 & 2.5380 & -2.9308 \\
\hline Manila, & 2.9000 & 1.8545 & 1.0455 \\
Philippines & 1.7518 & 1.1018 & 0.6501 \\
\hline Damascus, & -1.4058 & 6.2619 & -7.6677 \\
Syria & -1.7984 & 23.4284 & -25.2268 \\
\hline Average & 0.8611 & 5.9280 & -5.0669 \\
& 0.1912 & 5.9043 & -5.7130 \\
\hline
\end{tabular}

Table 3. Pitch/Roll Error Values for ERC

\begin{tabular}{|c|c|c|c|}
\hline Mission & $\begin{array}{c}\text { Pitch/Roll } \\
\text { Error } \\
\text { (Act - Prog) }\end{array}$ & $\begin{array}{c}\text { Pitch/Roll } \\
\text { Error } \\
\text { (Act - Proj) }\end{array}$ & $\begin{array}{c}\text { Pitch/Roll } \\
\text { Error } \\
\text { (Proj - Prog) }\end{array}$ \\
\hline $\begin{array}{c}\text { Tripoli, } \\
\text { Lebanon }\end{array}$ & $\begin{array}{c}1.6092 \\
0.7095\end{array}$ & $\begin{array}{l}8.2436 \\
1.3709\end{array}$ & $\begin{array}{l}-6.6343 \\
-0.6614\end{array}$ \\
\hline Dubai, UAE & 1.5195 & 7.0348 & -5.5154 \\
\hline
\end{tabular}




\begin{tabular}{|c|c|c|c|}
\hline & 0.5722 & 0.9686 & -0.3963 \\
\hline Muscat, & -0.3175 & 6.2329 & -6.5504 \\
Oman & -0.2568 & 2.5934 & -2.8502 \\
\hline Damascus, \\
Syria & -1.5383 & 6.2654 & -7.8037 \\
& -1.7584 & 23.4741 & -25.2326 \\
\hline Average & 0.3182 & 6.9442 & -6.6260 \\
& -0.1834 & 7.1017 & -7.2851 \\
\hline
\end{tabular}

Table 4. Pitch/Roll Error Values for HPT-R

\begin{tabular}{|c|c|c|c|}
\hline $\begin{array}{c}\text { Average } \\
\text { Pitch \& Roll }\end{array}$ & (Act - Prog) & (Act - Proj) & (Proj - Prog) \\
\hline & 0.5897 & 6.4361 & -5.8465 \\
Diwata-2 & 0.0039 & 6.5030 & -6.4991 \\
\hline
\end{tabular}

Table 5. Pitch/Roll averages for Diwata-2

Using the program that we developed, we were able to obtain the attitude of the satellite sensor on the different missions. It is noticeable that the error between the programmed target and the resulting image center is much smaller than the error between the projected target and the resulting image center. It is also worth noting that the error between the projected target and the actual image center is quite large. The large difference is due to measurement errors from the satellite's attitude sensor sources in the attitude logs. With a timing and pointing error of $8.09 \mathrm{~s}$ and $62.05 \mathrm{~km}$, respectively, for the ERC images and $9.49 \mathrm{~s}$ and $74.75 \mathrm{~km}$ for the HPT-R, there seem to be disturbances in the ACDS. Future studies may determine the cause of the large discrepancy.

Figures 1 to 5 show the position of the programmed and projected targets and the actual image centers of the ERC per area, together with the Diwata-2 position at the time of image capture. ERC images are used as the difference between the HPT-R and ERC image centers are relatively small. Figures 6 to 10 show the timing and pointing errors (from image center to programmed target) for the ERC images for each mission. The tabulated timing and pointing errors are in tables 6 and 7 for the ERC and the HPT-R, respectively. The direction of the Diwata2 in the captured images is downward along the path. Thus, the positive timing error values correspond to the error pointing to the direction of the Diwata-2 trajectory (thus downward). On the other hand, the positive pointing error values correspond to the error pointing to the right side of the trajectory (which on the image is on the left side).

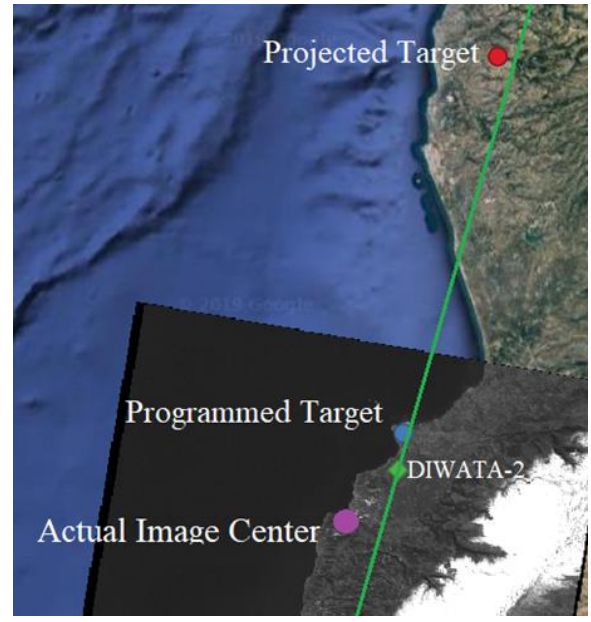

Figure 1. Tripoli, Lebanon. Relative positions. (C) Google Image Copyright 2019, CNES

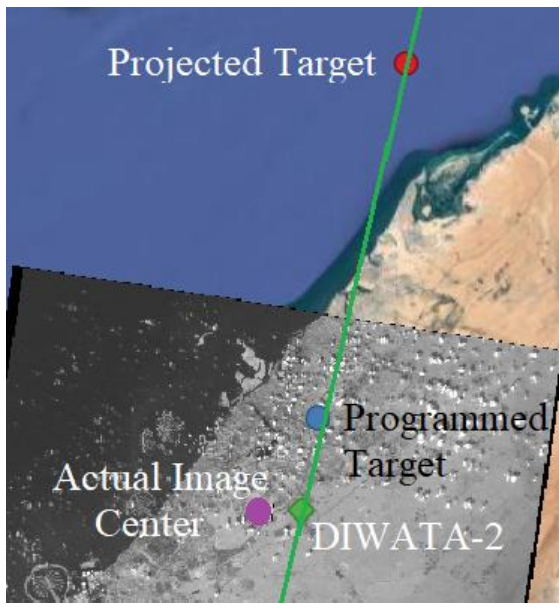

Figure 2. Dubai, UAE. Relative positions. C C Google Image Copyright 2019, Maxar

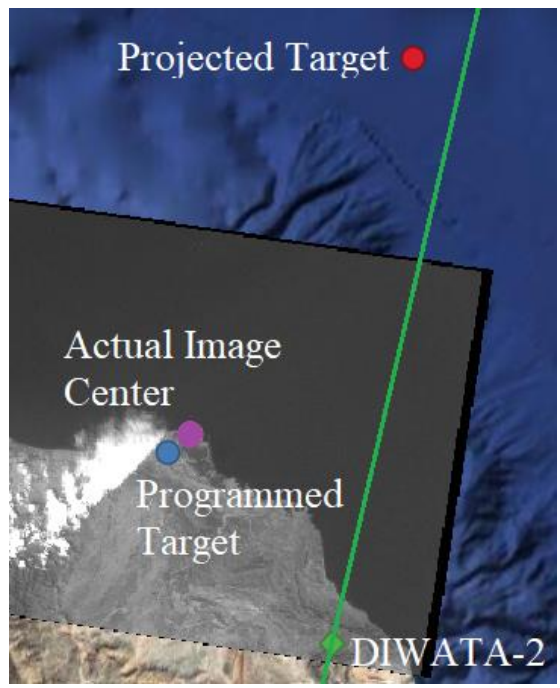

Figure 3. Muscat, Oman. Relative positions. (C) Google Image Copyright 2019, Maxar 


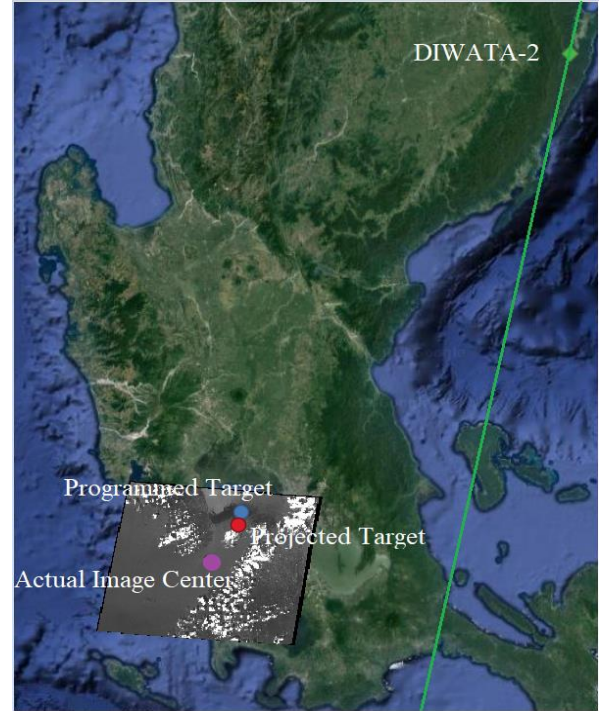

Figure 4. Manila, Philippines. Relative positions. (C) Google Image Copyright 2019, Maxar

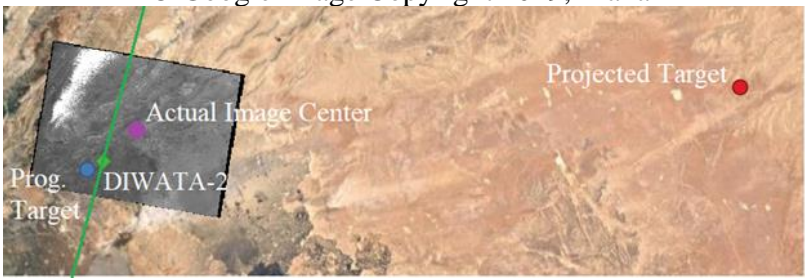

Figure 5. Damascus, Syria. Relative positions. (C) Google Image Copyright 2019, Maxar

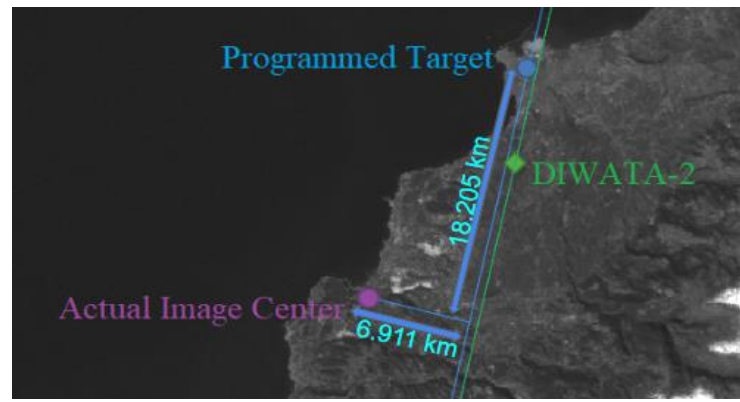

Figure 6. Tripoli, Lebanon. Distance from programmed target to actual image center.

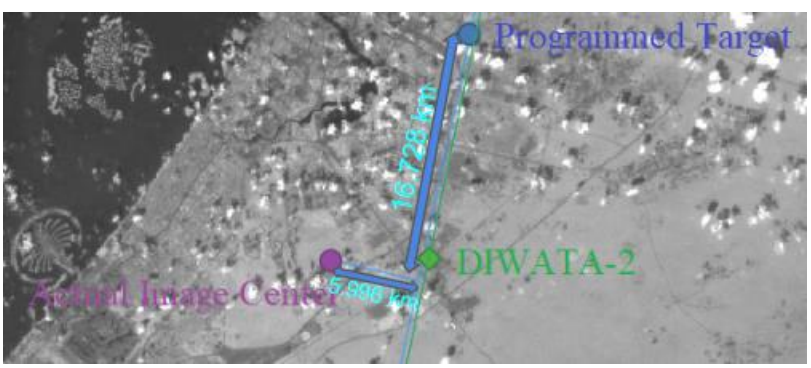

Figure 7. Dubai, UAE. Distance from programmed target to actual image center.

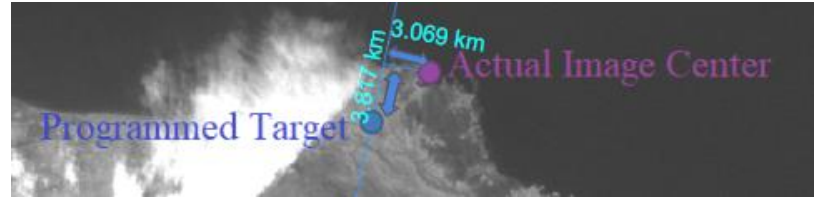

Figure 8. Muscat, Oman. Distance from programmed target to actual image center.

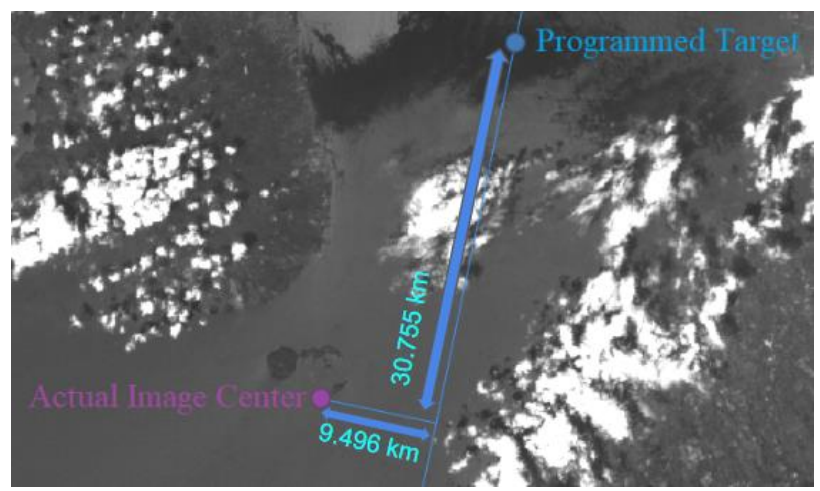

Figure 9. Manila, Philippines. Distance from programmed target to actual image center.

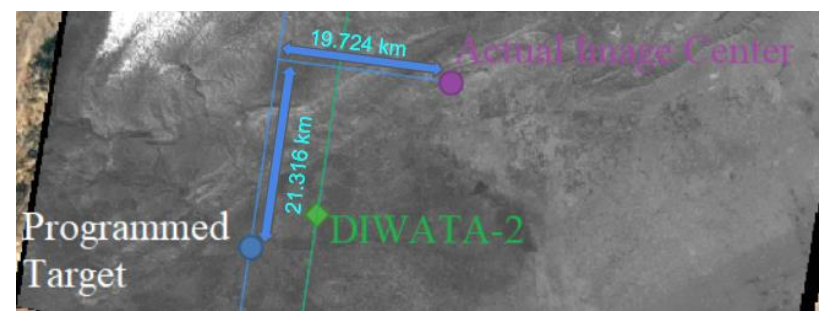

Figure 10. Damascus, Syria. Distance from programmed target to actual image center.

\begin{tabular}{|c|c|c|}
\hline Image (ERC) & $\begin{array}{c}\text { Timing Error } \\
(\mathrm{km})\end{array}$ & $\begin{array}{c}\text { Pointing Error } \\
(\mathrm{km})\end{array}$ \\
\hline Tripoli, Lebanon & 18.205 & 6.911 \\
\hline Dubai, UAE & 16.728 & 5.996 \\
\hline Muscat, Oman & -3.817 & -3.069 \\
\hline Manila, Philippines & 30.755 & 9.496 \\
\hline Damascus, Syria & -21.316 & -19.724 \\
\hline Average & 8.111 & -0.078 \\
\hline $\begin{array}{c}\text { Average (without the } \\
\text { Manila data) }\end{array}$ & 2.450 & -2.4715 \\
\hline
\end{tabular}

Table 6. Timing/Pointing Errors for ERC

\begin{tabular}{|c|c|c|}
\hline Image (HPT-R) & $\begin{array}{c}\text { Timing Error } \\
(\mathrm{km})\end{array}$ & $\begin{array}{c}\text { Pointing Error } \\
(\mathrm{km})\end{array}$ \\
\hline Tripoli, Lebanon & 17.901 & 6.187 \\
\hline Dubai, UAE & 16.466 & 5.411 \\
\hline
\end{tabular}




\begin{tabular}{|c|c|c|}
\hline Muscat, Oman & -3.446 & -2.447 \\
\hline Damascus, Syria & -22.597 & -19.485 \\
\hline Average & 2.081 & -2.583 \\
\hline
\end{tabular}

Table 7. Timing/Pointing Errors for HPT-R

\begin{tabular}{|c|c|c|}
\hline $\begin{array}{c}\text { Average Pitch \& } \\
\text { Roll }\end{array}$ & $\begin{array}{c}\text { Timing Error } \\
(\mathrm{km})\end{array}$ & $\begin{array}{c}\text { Pointing Error } \\
(\mathrm{km})\end{array}$ \\
\hline Diwata-2 & 2.2655 & -2.5272 \\
\hline
\end{tabular}

Table 8. Timing/Pointing Errors for Diwata-2

For the ERC images, the average pitch and roll errors from the programmed target to the actual image center introduce a $1.05 \mathrm{~s}$ timing error and a $2.0 \mathrm{~km}$ pointing error, respectively. For the HPT-R images, its average timing and pointing errors are $0.27 \mathrm{~s}$ and $1.92 \mathrm{~km}$, respectively. These are almost acceptable errors, as it is near the swath of the HPT images ( $3 \mathrm{~km} \times 2 \mathrm{~km})$. However, this applies only to the average errors. Most of the data used has errors exceeding the HPT swath but cancels out due to the direction of the error. Therefore, the attitude errors would cause the satellite to miss its intended target, and the non-uniformity of error directions would make it hard to determine a single value to correct the said errors.

It is worth noting that the pitch and roll values computed by the program for the targets are not the same as the pitch and roll values apparently shown in the images where the Diwata-2's position is determined by the STK through TLE propagation. As an example, the computed pitch value for the programmed target in Tripoli, Lebanon shows a positive value, suggesting that the said target is found when the satellite tilts forward. This is not the case in the image where the said target is found when tilting backwards. This suggests a discrepancy between the STK positions, and the position and velocity vectors recorded by the satellite's GPS module. Further investigations would show that the pitch and roll error values (or the distances between targets) are still correct thus the discrepancy lies in the Diwata-2's position due to relative positioning. Future studies could expand on this.

Despite the discrepancy, the error values extracted through measurements are almost the same to the error values extracted through the transformation system. Therefore, a working transformation system for attitude determination has been created and can be used to determine attitude values for targets. Furthermore, pointing errors computed from the method are angle based and are not affected by the varying altitude of the satellite. Figure 11 shows the effects of varying altitude to the point angle required to compensate for errors from $2 \mathrm{~km}$ to $20 \mathrm{~km}$ of the satellite. As an example, altitude variation in the range of $10 \mathrm{~km}$ affects the pointing angle requirement to compensate for the pointing error of $20 \mathrm{~km}$ by $0.07^{\circ}$ which is around $700 \mathrm{~km}$ if translated to ground distance.

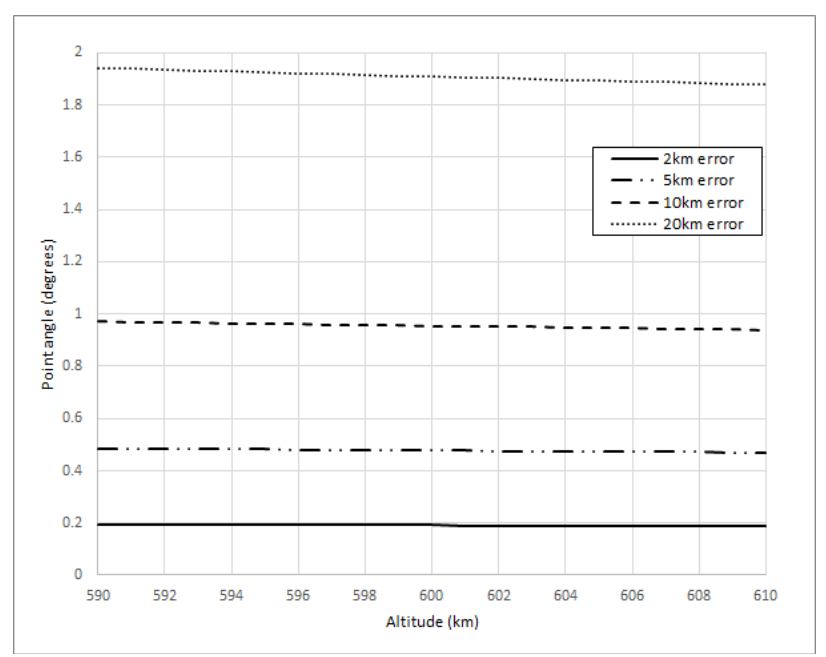

Figure 11. Pointing angle variation on different errors

\section{CONCLUSIONS}

Since a microsatellite does not undergo maintenance after launch, it is necessary to know its current health as future use considers the current status of the microsatellite. As in the case of Diwata-2, it is necessary to know the targeting errors so necessary adjustments can be made. Knowing that there is a disturbance in the ACDS would also lead to a better understanding of the satellite's current health and capabilities.

This study has created a system of transformation through the use of quaternions and thus determined the pitch and roll errors between the programmed target, predicted target and the actual center of the payloads. The use of quaternions in the transformation system simplified the equations used. As compared to using Euler angles and direction cosine matrices, quaternions can easily define coordinate transformation between multiple coordinate systems. Corrections derived from the results of the quaternion-based methods are not altitude dependent compared to corrections derived from computing the ground distance differences in latitude and longitude used on previous target assessments. The method fits Diwata-2 as its altitude varies by around $\pm 10 \mathrm{~km}$.

Although the computed values are small, these can still be adjusted when uploading targeting commands to provide a more exact image. However, careful calibration is recommended as the attitude errors do not seem to follow a definite pattern to be corrected. For attitude determination to improve, the position and velocity vectors of the satellite should be exactly known. Also, since these values are now known, future use of Diwata-2's targeting capabilities can be put to better use for other projects such as lunar calibration and change detection, which leads to better output of the satellite.

The transformation system created has discovered problems that can be studied in the future. Issues such as the Diwata-2's position and velocity vector discrepancies, and the issues in the satellite's recorded attitude values (thus, making the projected targets far from the actual image centers) need to be studied further in the future for possible causes and solutions. Also, since this study only considers the actual image centers of the camera products, other issues that change the position of the image center should be considered for future studies, such as relief displacement. 


\section{REFERENCES}

Aimati, N (Nurgul). "A Comparison of Rotation Parametrisations for Bundle Adjustment.” 2015.

Besl, Paul J., and Neil D. Mckay. "Method for Registration of 3D Shapes." Sensor Fusion IV: Control Paradigms and Data Structures, 1992, doi:10.1117/12.57955.

Groÿekatthöfer, Karsten, and Zizung Yoon. "Introduction into Quaternions for Spacecraft Attitude Representation." 31 May 2012, www.tuberlin.de/fileadmin/fg 169/miscellaneous/Quaternions.pdf.

Magallon, Benjamin Jonah P., et al. "Diwata-1 Target Pointing Error Assessment Using Orbit and Space Environment Prediction Model." 2018 IEEE International Conference on Aerospace Electronics and Remote Sensing Technology (ICARES), 2018, doi:10.1109/icares.2018.8547062.

Perumal, L. "Quaternion and Its Application in Rotation Using Sets of Regions.” 2011.

Rummel, R., and T. Peters. "Reference Systems in Satellite Geodesy.”2001.

Yang Y. "Spacecraft attitude determination and control.” 2011. 